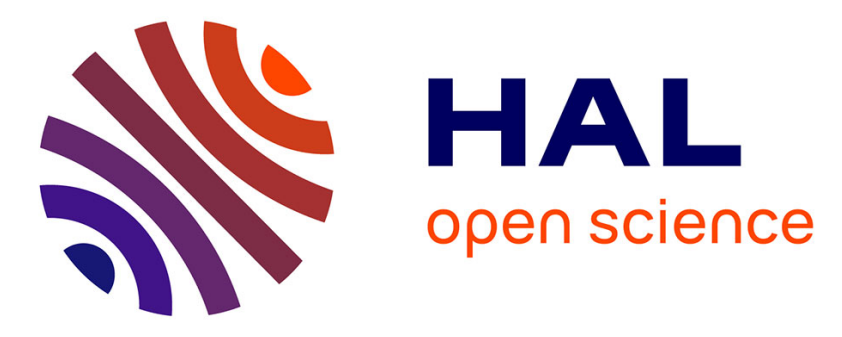

\title{
Delay-Dependent Positivity: Application to Interval Observers
}

Denis Efimov, Andrey Polyakov, Emilia Fridman, Wilfrid Perruquetti, Jean-Pierre Richard

\section{To cite this version:}

Denis Efimov, Andrey Polyakov, Emilia Fridman, Wilfrid Perruquetti, Jean-Pierre Richard. DelayDependent Positivity: Application to Interval Observers. Proc. ECC'15, Jul 2015, Linz, Austria. hal-01140336

\section{HAL Id: hal-01140336 \\ https://hal.inria.fr/hal-01140336}

Submitted on 8 Apr 2015

HAL is a multi-disciplinary open access archive for the deposit and dissemination of scientific research documents, whether they are published or not. The documents may come from teaching and research institutions in France or abroad, or from public or private research centers.
L'archive ouverte pluridisciplinaire HAL, est destinée au dépôt et à la diffusion de documents scientifiques de niveau recherche, publiés ou non, émanant des établissements d'enseignement et de recherche français ou étrangers, des laboratoires publics ou privés. 


\title{
Delay-Dependent Positivity: Application to Interval Observers
}

\author{
Efimov D., Polyakov A., Fridman E., Perruquetti W., Richard J.-P.
}

\begin{abstract}
New delay-dependent conditions of positivity for linear systems with time-varying delays are introduced. These conditions are applied to interval observer design for systems with time-varying delays in the state equations and in the measurements.
\end{abstract}

\section{INTRODUCTION}

An estimation in nonlinear delayed systems is rather complicated [1], [2], as well as analysis of functional differential equations [3]. Especially the observer synthesis is problematical for the cases when the model of a nonlinear delayed system contains parametric and/or signal uncertainties, or when the delay is time-varying or uncertain [4], [5], [6]. An observer solution for these more complex situations is highly demanded in many applications. Interval or set-membership estimation is a promising framework to observation in uncertain systems [7], [8], [9], [10], [11], [12], when all uncertainty is included in the corresponding intervals or polytopes, and as a result the set of admissible values (an interval) for the state is provided at each instant of time.

In this work an interval observer for time-delay systems is proposed. A peculiarity of an interval observer is that in addition to stability conditions, some restrictions on positivity of estimation error dynamics have to be imposed (in order to envelop the system solutions). The existing solutions in the field [13], [14], [15] are based on the delay-independent conditions of positivity from [16]. The first objective of this work is to introduce the delay-dependent positivity conditions, which are based on the theory of non-oscillatory solutions for functional differential equations [17]. Next, two designs of interval observers are given for linear systems with delayed measurements (with time-varying delays) for the case of observable and detectable systems.

The paper is organized as follows. Some preliminaries are given in Section 2. The delay-dependent positivity conditions are presented in Section 3. The interval observer design is performed for a class of linear time-delay systems (or a

All authors except E. Fridman are with Non-A team @ INRIA - LNE, Parc Scientifique de la Haute Borne, 40 avenue Halley, 59650 Villeneuve d'Ascq, France and CRIStAL (UMR-CNRS 9189), Ecole Centrale de Lille, BP 48, Cité Scientifique, 59651 Villeneuve-d'Ascq, France.

E. Fridman is with School of Electrical Engineering, Tel-Aviv University, Tel-Aviv 69978, Israel.

D. Efimov is with Department of Control Systems and Informatics, University ITMO, 49 av. Kronverkskiy, 197101 Saint Petersburg, Russia.

This work was supported in part by Région Nord-Pas de Calais, by the Government of Russian Federation (Grant 074-U01), the Ministry of Education and Science of Russian Federation (Project 14.Z50.31.0031) and by Israel Science Foundation (grant No 754/10). class of nonlinear systems in the output canonical form) with delayed measurements in Section 4. Example of numerical simulation is presented in Section 5.

\section{PRELIMINARIES}

In the rest of the paper, the following notation will be used:

- $\mathbb{R}$ is the Euclidean space $\left(\mathbb{R}_{+}=\{\tau \in \mathbb{R}: \tau \geq 0\}\right)$, $\mathcal{C}_{\tau}^{n}=C\left([-\tau, 0], \mathbb{R}^{n}\right)$ is the set of continuous maps from $[-\tau, 0]$ into $\mathbb{R}^{n}$ for $n \geq 1 ; \mathcal{C}_{\tau+}^{n}=\left\{y \in \mathcal{C}_{\tau}^{n}: y(s) \in\right.$ $\left.\mathbb{R}_{+}^{n}, s \in[-\tau, 0]\right\}$

- $x_{t}$ is an element of $\mathcal{C}_{\tau}^{n}$ associated with a map $x_{t}: \mathbb{R} \rightarrow$ $\mathbb{R}^{n}$ by $x_{t}(s)=x(t+s)$ for all $s \in[-\tau, 0]$

- $|x|$ denotes the absolute value of $x \in \mathbb{R},\|x\|$ is the Euclidean norm of a vector $x \in \mathbb{R}^{n},\|\varphi\|=$ $\sup _{t \in[-\tau, 0]}|\varphi(t)|$ for $\varphi \in \mathcal{C}_{\tau}$;

- for a measurable and locally essentially bounded input $u: \mathbb{R}_{+} \rightarrow \mathbb{R}^{p}$ the symbol $\|u\|_{\left[t_{0}, t_{1}\right]}$ denotes its $L_{\infty}$ norm $\|u\|_{\left[t_{0}, t_{1}\right]}=$ ess $\sup \left\{\|u(t)\|, t \in\left[t_{0}, t_{1}\right]\right\}$, or simply $\|u\|$ if $t_{0}=0$ and $t_{1}=+\infty$, the set of all such inputs $u \in \mathbb{R}^{p}$ with the property $\|u\|<\infty$ will be denoted as $\mathcal{L}_{\infty}^{p}$;

- for a matrix $A \in \mathbb{R}^{n \times n}$ the vector of its eigenvalues is denoted as $\lambda(A)$;

- $I_{n}$ and $0_{n \times m}$ denote the identity and zero matrices of dimensions $n \times n$ and $n \times m$ respectively;

- $a \mathcal{R} b$ corresponds to an elementwise relation $\mathcal{R}$ ( $a$ and $b$ are vectors or matrices): for example $a<b$ (vectors) means $\forall i: a_{i}<b_{i}$; for $\phi, \varphi \in \mathcal{C}_{\tau}$ the relation $\phi \mathcal{R} \varphi$ has to be understood elementwise for all domain of definition of the functions, i.e. $\phi(s) \mathcal{R} \varphi(s)$ for all $s \in[-\tau, 0]$;

- for a symmetric matrix $\Upsilon$, the relation $\Upsilon \prec 0(\Upsilon \preceq 0)$ means that the matrix is negative (semi) definite.

\section{A. General definition of positivity for time-delay systems}

Consider a linear non-autonomous functional differential equation

$$
\begin{gathered}
\dot{x}(t)=\sum_{k=1}^{m} A_{k}(t) x\left[h_{k}(t)\right]+b(t), t \geq t_{0} \geq 0, \\
x(t)=\phi(t) \text { for } t<t_{0}, x\left(t_{0}\right)=x_{0},
\end{gathered}
$$

where $x(t) \in \mathbb{R}^{n}$ is the state vector and $x_{0} \in \mathbb{R}^{n}$; all elements of $A_{k}$ are from $\mathcal{L}_{\infty}$ for all $1 \leq k \leq m, m>0$; the signals $h_{k}:\left[t_{0},+\infty\right) \rightarrow \mathbb{R}$ are Lebesgue measurable, $h_{k}(t) \leq t$ and $\lim _{t \rightarrow+\infty} h_{k}(t)=+\infty$ for all $1 \leq k \leq m$; 
$b \in \mathcal{L}_{\infty}^{n}$ is the input; $\phi:\left(-\infty, t_{0}\right) \rightarrow \mathbb{R}^{n}$ is a Borel measurable bounded function of initial conditions.

Definition 1. For each $s \geq 0$, the solution $\Upsilon(t, s) \in \mathbb{R}^{n \times n}$ of the problem

$$
\begin{gathered}
\frac{\partial \Upsilon(t, s)}{\partial t}=\sum_{k=1}^{m} A_{k}(t) \Upsilon\left[h_{k}(t), s\right], \\
\Upsilon(t, s)=0 \text { for } t<s, \Upsilon(s, s)=I_{n},
\end{gathered}
$$

is called the fundamental (or the Cauchy) matrix of (1).

Lemma 1. [17] (Lemma 9.1) For each $x_{0} \in \mathbb{R}^{n}$ and $\phi$ : $\left(-\infty, t_{0}\right) \rightarrow \mathbb{R}^{n}$ there exists a unique solution of (1), and it can be presented in the form

$$
\begin{aligned}
x(t) & =\Upsilon\left(t, t_{0}\right) x_{0}+\int_{t_{0}}^{t} \Upsilon(t, s) b(s) d s \\
& +\sum_{k=1}^{m} \int_{t_{0}}^{t} \Upsilon(t, s) A_{k}(s) \phi\left[h_{k}(s)\right] d s,
\end{aligned}
$$

where $\phi\left[h_{k}(s)\right]=0$ if $h_{k}(s)>t_{0}$.

Now we are in position to give the definition of positivity, denote $\Omega$ a subset of Borel measurable bounded functions $\phi:\left(-\infty, t_{0}\right) \rightarrow \mathbb{R}^{n}$.

Definition 2. The system (1) for $x_{0} \in \mathbb{R}_{+}^{n}$ and $b(t) \in \mathbb{R}_{+}^{n}$ is called $\Omega$-positive, if $x(t) \geq 0$ for all $t \geq t_{0}$ provided that $\phi \in \Omega$.

A direct consequence of this definition is that for $\Omega=\{0\}$ the system is $\{0\}$-positive (or we will write simply positive next) iff $\Upsilon(t, s) \in \mathbb{R}_{+}^{n \times n}$ for all $t \geq s$.

\section{B. Linear $\mathcal{C}_{\tau+}^{n}$-positive systems with constant delays}

Consider a linear system with a constant delay (a variant of (1) for $m=2$ )

$$
\begin{gathered}
\dot{x}(t)=A_{0} x(t)-A_{1} x(t-\tau)+b(t), t \geq t_{0} \geq 0, \\
x(\theta)=\phi(\theta) \text { for }-\tau \leq \theta \leq 0, \phi \in \mathcal{C}_{\tau}^{n},
\end{gathered}
$$

where $x(t) \in \mathbb{R}^{n}, x_{t} \in \mathcal{C}_{\tau}^{n}$ is the state function, $\tau \in \mathbb{R}_{+}$ is the delay; $b \in \mathcal{L}_{\infty}^{n}$ is the input; the constant matrices $A_{0}$ and $A_{1}$ have appropriate dimensions. The matrix $A_{0}$ is called Metzler if all its off-diagonal elements are nonnegative.

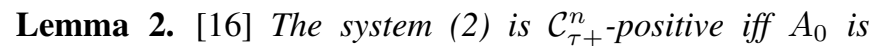
Metzler, $A_{1} \leq 0$ and $b(t) \in \mathbb{R}_{+}^{n}$ for all $t \in \mathbb{R} . A \mathcal{C}_{\tau+}^{n}$ positive system (2) is asymptotically stable for $b(t) \equiv 0$ for all $\tau \in \mathbb{R}_{+}$iff there are $p, q \in \mathbb{R}_{+}^{n}(p>0$ and $q>0)$ such that

$$
p^{T}\left[A_{0}-A_{1}\right]+q^{T}=0 .
$$

Under conditions of the above lemma the system has bounded solutions for $b \in \mathcal{L}_{\infty}^{n}$ with $b(t) \in \mathbb{R}_{+}^{n}$ for all $t \in \mathbb{R}$. Note that for linear time-invariant systems the conditions of positive invariance of polyhedral sets have been similarly given in [18], as well as conditions of asymptotic stability in the nonlinear case have been considered in [19], [20], [21].

\section{DELAY-DEPENDENT POSITIVITY}

Consider a scalar time-varying linear system with timevarying delays [17]:

$$
\begin{gathered}
\dot{x}(t)=a_{0}(t) x[g(t)]-a_{1}(t) x[h(t)]+b(t), \\
x(\theta)=0 \text { for } \theta<0,
\end{gathered}
$$

where $a_{0} \in \mathcal{L}_{\infty}, a_{1} \in \mathcal{L}_{\infty}, b \in \mathcal{L}_{\infty}, h(t)-t \in \mathcal{L}_{\infty}, g(t)-t \in$ $\mathcal{L}_{\infty}$ and $h(t) \leq t, g(t) \leq t$ for all $t \geq 0$. For the system (3) the initial condition in (4) is, in general, not a continuous function (if $x(0) \neq 0$ ).

The following result proposes delay-independent positivity conditions following Definition 2.

Lemma 3. [17] (Corollary 15.7) Let $h(t) \leq g(t)$ and $0 \leq$ $a_{1}(t) \leq a_{0}(t)$ for all $t \geq 0$. If $x(0) \geq 0$ and $b(t) \geq 0$ for all $t \geq 0$, then the corresponding solution of (3),(4) $x(t) \geq 0$ for all $t \geq 0$.

Recall that in this case positivity is guaranteed for "discontinuous" initial conditions and time-varying delays. The peculiarity of the condition $0 \leq a_{1}(t) \leq a_{0}(t)$ is that it may correspond to an unstable system (3). In order to overcome this issue, delay-dependent conditions can be introduced.

Lemma 4. [17] (Corollary 15.9) Let $h(t) \leq g(t)$ and $0 \leq$ $\frac{1}{e} a_{0}(t) \leq a_{1}(t)$ for all $t \geq 0$ with $(e=\exp (1))$

$$
\sup _{t \in \mathbb{R}_{+}} \int_{h(t)}^{t}\left[a_{1}(\xi)-\frac{1}{e} a_{0}(\xi)\right] d \xi<\frac{1}{e} .
$$

If $x(0) \geq 0$ and $b(t) \geq 0$ for all $t \geq 0$, then the corresponding solution of (3), (4) $x(t) \geq 0$ for all $t \geq 0$.

These lemmas describe positivity conditions in the sense of Definition 2 for the system (3), (4), which is more complex than (2), but scalar.

Corollary 1. [17] (Theorem 15.23) Consider the autonomous system (3) with $a_{0}(t)=a_{0}, a_{1}(t)=a_{1}, h(t)=t-\tau$ and $g(t)=t-\sigma$ with $0 \leq \sigma<\tau$. Let $0 \leq a_{0} \leq e a_{1}<a_{0}+\tau^{-1}$. If $x(0) \geq 0$ and $b(t) \geq 0$ for all $t \geq 0$, then the corresponding solution of (3), (4) $x(t) \geq 0$ for all $t \geq 0$.

Corollary 2. The system

$$
\begin{gathered}
\dot{x}(t)=A_{0} x(t)-A_{1} x(t-\tau)+b(t), t \geq t_{0} \geq 0, \\
x(\theta)=0 \text { for }-\tau \leq \theta<0, x(0) \in \mathbb{R}_{+}^{n},
\end{gathered}
$$

is positive if $-A_{1}$ is Metzler, $A_{0} \geq 0$,

$$
0 \leq\left(A_{0}\right)_{i, i} \leq e\left(A_{1}\right)_{i, i}<\left(A_{0}\right)_{i, i}+\tau^{-1}
$$

for all $i=1, \ldots, n$, and $b(t) \geq 0$ for all $t \geq 0$.

From these corollaries it is easy to conclude that the delay-dependent case studied in Lemma 3 and Lemma 4 is crucially different from the delay-independent $\mathcal{C}_{\tau+}^{n}$-positivity conditions given first in Lemma 2, where in the scalar case the restriction $a_{1} \leq 0$ implies positivity of (2) and the condition $a_{0}<a_{1}$ according to Lemma 2 ensures stability for any delay $\tau$. These results do not contradict to Remark 3.1 of [16], since $x(\theta) \neq 0$ for $-\tau \leq \theta<0$ there. A graphical 


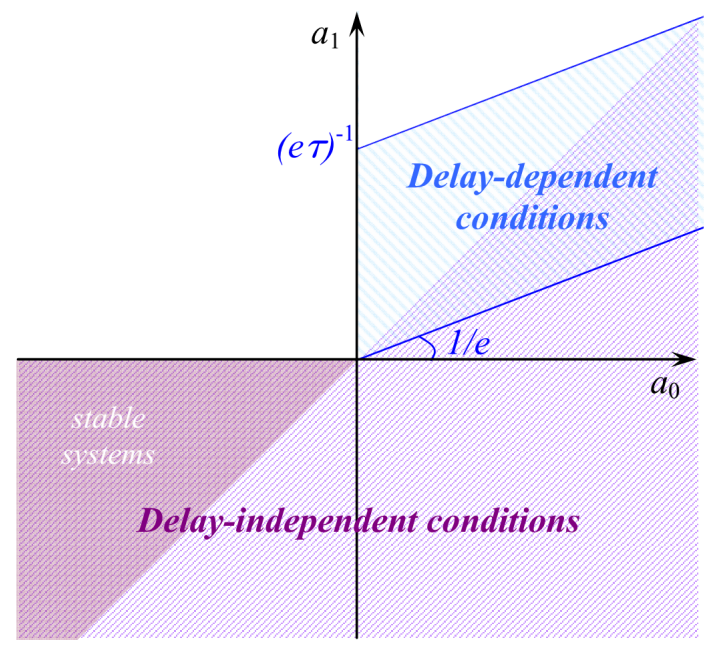

Figure 1. Different positivity conditions for (3)

illustration of different delay-independent conditions $\left(\mathcal{C}_{\tau+}^{n}\right.$ positivity from Lemma 2 and positivity from Lemma 3 ) and delay-dependent ones (from Lemma 4, the stability conditions are also satisfied in this case) for the system (3) is given in Fig. 1 in the plane $\left(a_{0}, a_{1}\right)$. It is worth stressing that an extension of the positivity domain in Lemma 4 is also achieved due to restrictions imposed on initial conditions in (4).

In order to use the results of Lemma 3 and Lemma 4 it is necessary to pass from discontinuous initial conditions in (4) to continuous ones usually studied [1]. In this section we will be interested in the case

$$
a_{0}(t)=a_{0}, a_{1}(t)=a_{1}, t-\bar{\tau} \leq h(t) \leq t-\underline{\tau}, g(t)=t,
$$

where $\bar{\tau} \geq \underline{\tau} \geq 0$ are minimum and maximum delays respectively. Then the following corollary of Lemma 4 can be formulated.

Corollary 3. Let $0 \leq a_{0} \leq e a_{1}<a_{0}+\bar{\tau}^{-1}$. If $x(0) \geq 0$ and $b(t) \geq 0$ for all $t \geq 0$, then the corresponding solution of (3), (5), (4) $x(t) \geq 0$ for all $t \geq 0$.

Remark 1. As mentioned in [22], the first delay interval $0 \leq$ $t \leq \bar{\tau}$ is important for delay-dependent conditions giving solution bounds (and not just stability conditions).

Now let us substitute the initial condition (4) with a continuous one:

$$
x(\theta)=\phi(\theta) \text { for } \theta \in[-\bar{\tau}, 0],
$$

and consider the conditions providing delay-dependent $\mathcal{C}_{\tau+}^{n}{ }^{-}$ positivity for (3), (5), (6).

Proposition 1. Let $0 \leq a_{0} \leq e a_{1}<a_{0}+\bar{\tau}^{-1}$. If $x(0) \geq 0$, $b(t) \geq 0$ for all $t \geq 0$ and

$$
b(t) \geq a_{1} \phi[h(t)] \quad \forall t \in\{0 \leq t \leq \bar{\tau}: h(t)<0\},
$$

then the corresponding solution of (3), (5), (6) satisfies $x(t) \geq 0$ for all $t \geq 0$.

All proofs are excluded due to space limitations.
Let us show how these conditions can be used for the design of interval observers.

\section{INTERVAL OBSERVER DESIGN UNDER DELAYED MEASUREMENTS}

In this section a useful inequality for interval analysis and a statement of the problem are given. Next, a motivating benchmark example from [13] is investigated, using the results of the previous section, in order to clarify the main idea. Finally, a delay-dependent approach for an interval observer design is presented.

\section{A. Interval bounds}

Given a matrix $A \in \mathbb{R}^{m \times n}$ define $A^{+}=\max \{0, A\}$, $A^{-}=A^{+}-A$ and $|A|=A^{+}+A^{-}$. Let $x \in \mathbb{R}^{n}$ be a vector variable, $\underline{x} \leq x \leq \bar{x}$ for some $\underline{x}, \bar{x} \in \mathbb{R}^{n}$, and $A \in \mathbb{R}^{m \times n}$ be a constant matrix, then

$$
A^{+} \underline{x}-A^{-} \bar{x} \leq A x \leq A^{+} \bar{x}-A^{-} \underline{x} .
$$

This claim follows from the equation $A x=\left(A^{+}-A^{-}\right) x$, that for $\underline{x} \leq x \leq \bar{x}$ gives the required estimates.

\section{B. Problem statement}

Consider a linear system with a time-varying delay:

$$
\begin{aligned}
\dot{x}(t) & =A_{0} x(t)+A_{1} x[h(t)]+b(t), \\
y(t) & =C x[h(t)]+v(t),
\end{aligned}
$$

where $x(t) \in \mathbb{R}^{n}, t-\bar{\tau} \leq h(t) \leq t-\underline{\tau}$ is a known timevarying delay $\left(t-h(t) \in \mathcal{L}_{\infty}\right), 0 \leq \underline{\tau} \leq \bar{\tau}$ are minimum and maximum delays respectively, $x_{0} \in \mathcal{C}_{\bar{\tau}} ; y(t) \in \mathbb{R}^{p}$ is the system output available for measurements with the noise $v \in \mathcal{L}_{\infty}^{p} ; b \in \mathcal{L}_{\infty}^{n}$ is the system input; the constant matrices $A_{0}, A_{1}$ and $C$ have appropriate dimensions. It is assumed that for given $b$ and $h$ the system has a unique solution defined at least locally.

Assumption 1. For given input $b(t)$, delay $h(t)$ and initial condition $x_{0} \in \mathcal{C}_{\bar{\tau}}$, the corresponding solution of (8) $x(t)$ is bounded. In addition, there exist known functions $\underline{x}_{0}, \bar{x}_{0} \in \mathcal{C}_{\bar{\tau}}$ such that $\underline{x}_{0}(\theta) \leq x_{0}(\theta) \leq \bar{x}_{0}(\theta)$ for all $\theta \in[-\bar{\tau}, 0]$.

Boundedness of the state $x(t)$ is a usual assumption in the estimation theory [23], [24]. The assumption about a known set $\left[\underline{x}_{0}, \bar{x}_{0}\right]$ for the initial conditions $x_{0}$ is standard for the interval or set-membership estimation theory [14], [7], [8], [9], [10]. We will assume that the values of matrices $A_{0}$, $A_{1}$ and $C$ are known, for the uncertain delay $h(t)$ only the bounds $\underline{\tau}, \bar{\tau}$ are given, the instant values of the signals $b(t)$ and $v(t)$ are unavailable.

Assumption 2. There exist known signals $\underline{b}, \bar{b} \in \mathcal{L}_{\infty}^{n}$ and $\underline{v}, \bar{v} \in \mathcal{L}_{\infty}^{p}$ such that $\underline{b}(t) \leq b(t) \leq \bar{b}(t)$ and $\underline{v}(t) \leq v(t) \leq$ $\bar{v}(t)$ for all $t \geq 0$.

Therefore, the uncertain inputs $b(t), h(t)$ and $v(t)$ in (8) belong to known intervals $[\underline{b}(t), \bar{b}(t)],[t-\bar{\tau}, t-\underline{\tau}]$ and $[\underline{v}(t), \bar{v}(t)]$ respectively for all $t \geq 0$. 
It is required to design an interval observer,

$$
\begin{gathered}
\dot{\xi}(t)=F\left[\xi_{t}, \underline{b}(t), \bar{b}(t), \underline{v}(t), \bar{v}(t), y(t)\right], \xi_{t} \in \mathcal{C}_{\bar{\tau}}^{s}, \\
\underline{x}(t)=\underline{G}\left[\xi_{t}, \underline{b}(t), \bar{b}(t), \underline{v}(t), \bar{v}(t), y(t)\right], \\
\bar{x}(t)=\bar{G}\left[\xi_{t}, \underline{b}(t), \bar{b}(t), \underline{v}(t), \bar{v}(t), y(t)\right]
\end{gathered}
$$

such that $\underline{x}(t) \leq x(t) \leq \bar{x}(t)$ for all $t>0$ provided that $\underline{x}_{0} \leq x_{0} \leq \bar{x}_{0}$, and $\underline{x}, \bar{x} \in \mathcal{L}_{\infty}^{n}, s>0$. A similar problem has been studied in [13] but for constant delays.

\section{Motivating example}

Consider a motivating example introduced in [13], where the problem of a framer design (the definition of a framer can be found in [13], roughly speaking it is an interval open-loop estimator independent of $y(t))$ has been posed for a scalar system

$$
\dot{x}(t)=-x(t-\tau)
$$

with initial condition $x_{0} \in \mathcal{C}_{\tau}$. This system is globally asymptotically stable if $\tau<\frac{\pi}{2}$. It has been proven in [13] that this system has no framer of the form

$$
\begin{gathered}
F\left(\xi_{t}\right)=F_{1} \xi(t)+F_{2} \xi(t-\tau), \\
\underline{G}\left(\xi_{t}\right)=H_{1} \xi(t), \bar{G}\left(\xi_{t}\right)=H_{2} \xi(t),
\end{gathered}
$$

where $F_{i}, H_{i}(i=1,2)$ are matrices of appropriate dimensions.

Applying the result of Proposition 1 or Corollary 1, the system (9) has positive solutions for a discontinuous initial condition (4) with $x(0) \geq 0$ if $\tau<\frac{1}{e}$. Actually in this case it has a non-oscillating solution which is asymptotically converging to zero (since $\frac{1}{e}<\frac{\pi}{2}$ ), and which does not cross the zero level for all $t \in \mathbb{R}_{+}$. Further, using the result of Proposition 1, we can design a framer for (9) of a form similar to (10).

Claim 1. For the system (9) with any initial condition $x_{0} \in$ $\mathcal{C}_{\tau}$ and $\tau<\frac{1}{e}$, the system

$$
\begin{aligned}
& \underline{\dot{x}}(t)=-\underline{x}(t-\tau)+\delta\left(\left\|\underline{x}_{0}\right\|\right), \\
& \dot{\bar{x}}(t)=-\bar{x}(t-\tau)+\delta\left(\left\|\bar{x}_{0}\right\|\right), \\
& \delta(s)= \begin{cases}s & \text { if } t \leq \tau, \\
0 & \text { otherwise }\end{cases}
\end{aligned}
$$

is a framer, i.e. $\underline{x}(t) \leq x(t) \leq \bar{x}(t)$ for all $t>0$, provided that $\underline{x}_{0} \leq x_{0} \leq \bar{x}_{0}$, and $\underline{x}, \bar{x} \in \mathcal{L}_{\infty}^{n}$.

Therefore, a framer of a form similar to (10) can be designed for (9) with a restricted value of delay $\tau<\frac{1}{e}$ (it differs from (10) only on the interval $[0, \tau]$ ). The results of simulation for this framer are given in Section 5.

Let us extend this idea of interval observer design to a more generic system (8).

\section{Delay-dependent conditions for interval estimation}

The equation (8) can be rewritten as follows: $\dot{x}(t)=A_{0} x(t)+\left\{A_{1}-L C\right\} x[h(t)]+L y(t)+b(t)-L v(t)$, where $L \in \mathbb{R}^{n \times p}$ is an observer gain to be designed.
Assumption 3. There exists an invertible matrix $S \in \mathbb{R}^{n \times n}$ such that $S\left(A_{1}-L C\right) S^{-1}=R_{1}$, where $R_{1}$ is a Metzler matrix and

$$
R_{1}=R_{1}^{\dagger}+R_{1}^{2}, R_{1}^{\dagger}=\operatorname{diag}\left[-r_{1,1}, \ldots,-r_{1, n}\right], R_{1}^{2} \geq 0
$$

with $R_{1}^{\dagger}$ is the diagonal matrix composed by all elements on the main diagonal of $R_{1}, r_{1, i}>0$ for all $i=1, \ldots, n$, and $R_{1}^{2}$ is formed by the rest elements of $R_{1}$ out of the main diagonal.

The conditions of existence of such matrices $S$ and $L$ can be found in [12], in particular Assumption 3 is satisfied if the pair $\left(A_{1}, C\right)$ is observable. Under this assumption in the new coordinates $z=S x$ the system (8) takes the form:

$$
\dot{z}(t)=R_{0} z(t)+R_{1} z[h(t)]+S L y(t)+\beta(t),
$$

where $R_{0}=S A_{0} S^{-1}$ and $\beta(t)=S[b(t)-L v(t)]$ is a new additive uncertain input, the initial condition $z_{0}=S x_{0} \in \mathcal{C}_{\bar{\tau}}$ and

$$
\underline{z}_{0} \leq z_{0} \leq \bar{z}_{0}
$$

where $\underline{z}_{0}=S^{+} \underline{x}_{0}-S^{-} \bar{x}_{0}$ and $\bar{z}_{0}=S^{+} \bar{x}_{0}-S^{-} \underline{x}_{0}$ are calculated using (7). From Assumption 2 and the relations (7) we obtain that

$$
\underline{\beta}(t) \leq \beta(t) \leq \bar{\beta}(t) \quad \forall t \geq 0,
$$

where $\beta(t)=S^{+} \underline{b}(t)-S^{-} \bar{b}(t)-(S L)^{+} \bar{v}(t)+(S L)^{-} \underline{v}(t)$ and $\bar{\beta}(t)=S^{+} \bar{b}(t)-S^{-} \underline{b}(t)-(S L)^{+} \underline{v}(t)+(S L)^{-} \bar{v}(t)$. Then the following interval observer can be proposed for the representation (11):

$$
\begin{aligned}
& \underline{\dot{z}}(t)=R_{0}^{+} \underline{z}(t)-R_{0}^{-} \bar{z}(t)+R_{1} \underline{z}[h(t)] \\
& +S L y(t)+\underline{\beta}(t)-\delta \text {, } \\
& \dot{\bar{z}}(t)=R_{0}^{+} \bar{z}(t)-R_{0}^{-} \underline{z}(t)+R_{1} \bar{z}[h(t)] \\
& +S L y(t)+\bar{\beta}(t)+\delta \text {, } \\
& \delta=\left[\delta_{1}, \ldots, \delta_{n}\right]^{\mathrm{T}} \text {, } \\
& \delta_{i}=\left\{\begin{array}{ll}
r_{1, i}\left\|\bar{z}_{0, i}-\underline{z}_{0, i}\right\| & \text { if } t \leq \bar{\tau} \\
0 & \text { otherwise }
\end{array}, i=1, \ldots n\right.
\end{aligned}
$$

with initial conditions $\underline{z}_{0}, \bar{z}_{0}$ for the variables $\underline{z}(t), \bar{z}(t)$ respectively. Finally interval estimates for the variable $x(t)$ can also be obtained using (7):

$$
\begin{aligned}
& \underline{x}(t)=\left(S^{-1}\right)^{+} \underline{z}(t)-\left(S^{-1}\right)^{-} \bar{z}(t), \\
& \bar{x}(t)=\left(S^{-1}\right)^{+} \bar{z}(t)-\left(S^{-1}\right)^{-} \underline{z}(t) .
\end{aligned}
$$

For all $i=1, \ldots, n$ denote

$$
r_{0, i}=\left(R_{0}^{+}\right)_{i, i}
$$

Theorem 1. Let assumptions 1-3 be satisfied and

$$
r_{0, i} \leq e r_{1, i}<r_{0, i}+\bar{\tau}^{-1}
$$

for all $i=1, \ldots, n$. Then for the system (8) and the interval observer (12), (13)

$$
\underline{x}(t) \leq x(t) \leq \bar{x}(t) \quad \forall t \geq 0 .
$$




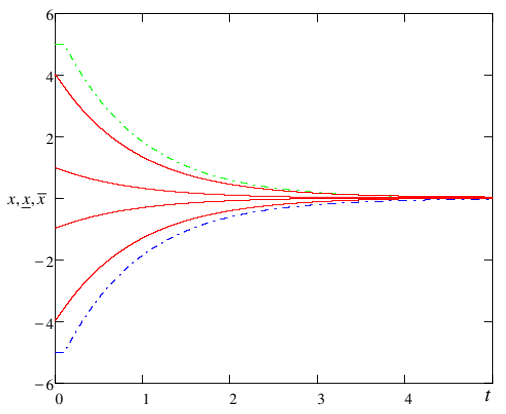

Figure 2. The results of simulation for the motivating example

If in addition there exist symmetric matrices $P \in \mathbb{R}^{2 n \times 2 n}$, $\sum_{L M I S} \in \mathbb{R}^{2 n \times 2 n}, \Xi \in \mathbb{R}^{2 n \times 2 n}$ and $\Theta \in \mathbb{R}^{2 n \times 2 n}$ such that the

$$
\left[\begin{array}{cccc}
\Phi_{0}^{T} P+P \Phi_{0}+\Sigma-\Xi & {\left[\begin{array}{cc}
\Xi & \Theta \\
\Theta & \Xi
\end{array}\right] \succeq 0,} & \\
\Theta & \Theta & P \Phi_{1}+\Xi-\Theta & \bar{\tau} \Phi_{0}^{T} \Xi \\
\Phi_{1}^{T} P+\Xi-\Theta & -\Sigma-\Xi & \Xi-\Theta & 0_{2 n \times 2 n} \\
\bar{\tau} \Xi \Phi_{0} & \Xi-\Theta & \Theta+\Theta^{T}-2 \Xi & \bar{\tau} \Phi_{1}^{T} \Xi \\
0_{2 n \times 2 n} & \bar{\tau} \Xi \Phi_{1} & -\Xi
\end{array}\right] \prec 0
$$

where

$$
\Phi_{0}=\left[\begin{array}{cc}
R_{0}^{+} & -R_{0}^{-} \\
-R_{0}^{-} & R_{0}^{+}
\end{array}\right], \Phi_{1}=\left[\begin{array}{cc}
R_{1} & 0_{n \times n} \\
0_{n \times n} & R_{1}
\end{array}\right],
$$

are satisfied, then $\underline{x}, \bar{x} \in \mathcal{L}_{\infty}^{n}$.

Let us illustrate this result by numerical simulations.

\section{FRAMER FOR THE MOTIVATING EXAMPLE}

The results of simulation of the framer from Claim 1 for the system (9) are shown in Fig. 2. For simulation the value $\tau=0.1<\frac{1}{e}$ and it has been assumed that $\left\|x_{0}\right\| \leq 5$. The red solid curves represent trajectories of (9) for different initial conditions, the blue and green dash-dot lines correspond to the the interval estimates $\underline{x}(t)$ and $\bar{x}(t)$ generated by the framer. As we can see, in this case the additional term $\delta$ "freezes" the estimates $\underline{x}(t), \bar{x}(t)$ on the interval $t \in[0, \tau]$, next they are converging to zero.

\section{CONCLUSION}

In the paper, new delay-dependent positivity conditions for linear systems with time-varying delays have been proposed. These conditions are related with non-oscillatory behavior of solutions [17]. They nicely complement the existing delayindependent conditions of [16] (see Fig. 1). Next, these new conditions have been employed to design interval observers for the systems with delayed measurements extending the theory of [13], [14], [15]. The results have been applied for the benchmark system from [13]. Extension of these results for the case of sampled-data measurements is a direction of future research.

\section{REFERENCES}

[1] R. Sipahi, S.-I. Niculescu, C. Abdallah, W. Michiels, and K. Gu, "Stability and stabilization of systems with time delay limitations and opportunities," IEEE Control Systems Magazine, vol. 31, no. 1, pp. 38-65, 2011.
[2] E. Fridman, Introduction to Time-Delay Systems: Analysis and Control. Basel: Birkhäuser, 2014.

[3] J.-P. Richard, "Time delay systems: an overview of some recent advances and open problems," Automatica, vol. 39, no. 10, pp. 16671694, 2003.

[4] C. Briat, O. Sename, and J.-F. Lafay, "Design of LPV observers for LPV time-delay systems: an algebraic approach," Int. J. Control, vol. 84, no. 9, pp. 1533-1542, 2011.

[5] C. Califano, L. Marquez-Martinez, and C. Moog, "On the observer canonical form for nonlinear time-delay systems," in Proc. 18th IFAC World Congress, Milano, 2011.

[6] G. Zheng, J.-P. Barbot, D. Boutat, T. Floquet, and J.-P. Richard, "On observation of time-delay systems with unknown inputs," IEEE Trans. Automatic Control, vol. 56, no. 8, pp. 1973-1978, 2011.

[7] J. Gouzé, A. Rapaport, and M. Hadj-Sadok, "Interval observers for uncertain biological systems," Ecological Modelling, vol. 133, pp. 46$56,2000$.

[8] L. Jaulin, "Nonlinear bounded-error state estimation of continuous time systems," Automatica, vol. 38, no. 2, pp. 1079-1082, 2002.

[9] M. Kieffer and E. Walter, "Guaranteed nonlinear state estimator for cooperative systems," Numerical Algorithms, vol. 37, pp. 187-198, 2004.

[10] M. Moisan, O. Bernard, and J. Gouzé, "Near optimal interval observers bundle for uncertain bio-reactors," Automatica, vol. 45, no. 1, pp. $291-$ $295,2009$.

[11] F. Mazenc and O. Bernard, "Interval observers for linear time-invariant systems with disturbances," Automatica, vol. 47, no. 1, pp. 140-147, 2011.

[12] T. Raïssi, D. Efimov, and A. Zolghadri, "Interval state estimation for a class of nonlinear systems," IEEE Trans. Automatic Control, vol. 57, no. 1 , pp. 260-265, 2012.

[13] F. Mazenc, S. Niculescu, and O. Bernard, "Exponentially stable interval observers for linear systems with delay," SIAM J. Control Optim., vol. 50, no. 1, pp. 286-305, 2012.

[14] D. Efimov, W. Perruquetti, and J.-P. Richard, "Interval estimation for uncertain systems with time-varying delays," International Journal of Control, vol. 86, no. 10, pp. 1777-1787, 2013.

[15] D. Efimov, A. Polyakov, and J.-P. Richard, "Interval estimation for systems with time delays and algebraic constraints," in Proc. European Control Conference (ECC), Strasbourg, 2014.

[16] W. Haddad and V. Chellaboina, "Stability theory for nonnegative and compartmental dynamical systems with time delay," Syst. Control Letters, vol. 51, pp. 355-361, 2004.

[17] R. P. Agarwal, L. Berezansky, E. Braverman, and A. Domoshnitsky, Nonoscillation theory of functional differential equations with applications. New York: Springer, 2012.

[18] M. Dambrine, J. Richard, and P. Borne, "Feedback control of timedelay systems with bounded control and state," Mathematical Problems in Engineering, vol. 1, no. 1, pp. 77-87, 1995.

[19] M. Dambrine and J. Richard, "Stability analysis of time-delay systems," Dynamic Systems and Applications, vol. 2, no. 3, pp. 405-414, 1993.

[20] — "Stability and stability domains analysis for nonlinear differential-difference equations," Dynamic Systems and Applications, vol. 3, no. 3, pp. 369-378, 1994.

[21] P. Borne, M. Dambrine, W. Perruquetti, and J.-P. Richard, Stability Theory at the end of the XXth Century. London: Taylor \& Francis, 2003, ch. Vector Lyapunov Function: Nonlinear, Time-Varying, Ordinary and Functional Differential Equations, pp. 49-73.

[22] K. Liu and E. Fridman, "Delay-dependent methods and the first delay interval," Systems \& Control Letters, vol. 64, pp. 57-63, 2014.

[23] G. Besançon, Ed., Nonlinear Observers and Applications, ser. Lecture Notes in Control and Information Sciences. Springer, 2007, vol. 363.

[24] T. Fossen and H. Nijmeijer, New Directions in Nonlinear Observer Design. Springer, 1999. 